\title{
Evaluation Regarding the Efficacy and Safety of a Probiotic Mixture in Healthy Volunteers with Evacuation Disorders
}

\author{
Lourdes Rodríguez-Fragoso ${ }^{1}$, Adriana Sandoval-Ocampo ${ }^{1}$, Martha Corbalá-Nava ${ }^{1}$, \\ Carlos A. Arjona-Canul ${ }^{1}$, Diana Lizbeth Gomez-Galicia ${ }^{1}$, Guillermo Tellez ${ }^{2}$, \\ Billy Hargis $^{2}$, Jorge Reyes-Esparza ${ }^{1}$
}

\author{
${ }^{1}$ Facultad de Farmacia, Universidad Autonoma del Estado de Morelos, Cuernavaca, Mexico; ${ }^{2}$ Department of Poultry Science, Uni- \\ versity of Arkansas, Fayetteville, USA. \\ Email: jareyes@uaem.mx
}

Received September 21 ${ }^{\text {st }}, 2011$; revised November $3^{\text {rd }}$, 2011; accepted November $12^{\text {th }}, 2011$

\begin{abstract}
Probiotics have been used in humans for almost a century and are widely recommended because they play an important role in gastrointestinal physiological and pathophysiological processes. The aim of this study was to evaluate the efficacy and safety of a probiotic mixture in healthy volunteers with evacuation disorders. We undertook a prospective study with historical control. We used the number of evacuations, effort and stool consistency 10 days before, 10 days during and 10 days after treatment with the probiotic as primary outcome measures. Eligible participants comprised 25 patients with an average age of 25.12 years (range: 18 - 55) who suffered from evacuation disorders. BIO L6 is a functional food consisting of a probiotic preparation that contains $1 \mathrm{mg}\left(10^{8}\right.$ CFUs) of lyophilized strains of Lactic Acid Bacteria resuspended in $250 \mathrm{~mL}$ of drinking water. The probiotic preparation was prescribed daily for two weeks, five days a week. A significant increase in the number of evacuations was observed in 23 patients during BIO L6 consumption and continued in 20 after consumption. The degree of effort spent defecating decreased in 17 patients during treatment and in 7 after treatment. A significant improvement $(P<0.001)$ in stool consistency was also observed in all patients receiving BIO L6. Fourteen patients had at least one adverse event (AE) for one or two days during treatment; most treatment-related AEs were considered minor or mild in terms of severity and were consistent with AEs reported for probiotic consumption. The results of this study suggest that the use of probiotic mixture BIO L6 is effective, safe and tolerable and provides beneficial effects for subjects with evacuations disorders.
\end{abstract}

Keywords: Chronic Constipation; Probiotic; Lactic Acid Bacteria

\section{Introduction}

The gastrointestinal tract is a complex ecosystem that hosts a diverse and highly evolved microbial community composed of hundreds of different microbial species [1]. The interactions between this complex community and the human host have become the focus of scientific research due to an increase in the incidence of illnesses related to deficient or compromised microflora (e.g., gastrointestinal tract infections, inflammatory bowel disease, irritable bowel syndrome, antibiotic-induced diarrhea, constipation, food allergies, cardiovascular disease, and certain cancers) [2]. The importance of intestinal microflora composition in physiological and pathophysiological processes in the gastrointestinal tract (GIT) has become increasingly evident and led to new preventive and therapeutic options [3-5]. It has been documented that primary motility disturbances can induce changes in intestinal microflora content and thereby further disturb intestinal physiology (and vice versa) [6].

Probiotics have been used in humans for almost a century and are widely recommended for the treatment of a variety of ills assumed to be of colonic origin, including diarrhea, constipation, bloating, and flatulence [7]. Probiotics may have potential beneficial effects in several gastroenteric conditions, especially when the intestinal flora has been disturbed [8]. New discoveries are specifically related to the beneficial effects of Lactic Acid Bacteria (LAB). It is possible to increase the proportion of LAB in the gastrointestinal microflora through probiotic consumption or oral administration of specific nondigestible substrates such as oligofructose [9]. Nowadays, there is a growing interest in the use of probiotics as a safe way of changing the intestinal microflora to treat gas- 
trointestinal diseases and, particularly, for modulating intestinal transit [10].

Evacuation disorders and hard feces are common in industrialized countries, affecting an average of $12 \%$ to $17 \%$ of the healthy adult population of any age [11]. Constipation not only interferes with quality of life, it also often ranks as a syndrome requiring competent medical intervention. Constipation relief is often mentioned as a potential health benefit of probiotics [8]. However, there have been relatively few studies in this field and the results have been conflicting. Some noted an increase in the number of stools and a reduction of symptoms in adult patients with chronic constipation, while others observed no improvement [12-16]. The effect of LAB ingestion on orofecal gut transit time appears to be dependent on the specific bacterial strain(s) used and the population being studied. Different probiotic strains might perform differently when treating constipation. The aim of this study was to evaluate the efficacy and safety of a probiotic mixture in healthy volunteers with evacuations disorders.

\section{Materials and Methods}

\subsection{Study Design}

This was a prospective study with historical control conducted on 25 healthy volunteers suffering evacuation disorders. Volunteers were students and workers from the College of Pharmacy at the Universidad Autónoma del Estado de Morelos in Cuernavaca, Morelos (Mexico). Subjects were enrolled after a complete physical examination, normal value of laboratory tests, and no evidence of gastrointestinal disease at plain abdomen $\mathrm{x}$-ray and ultrasound studies. Exclusion criteria were: constipation caused by enteric neuromuscular, anatomic, or metabolic diseases (as established by medical history, an abnormal thyroid hormone level, or prior anorectal manometry, barium, or ionogram examination); clinical signs of coexisting acute systemic illnesses; underlying severe chronic diseases; food allergies or other chronic gastrointestinal diseases; use of probiotics (yogurt) and prebiotics in the previous two weeks, or use of antibiotics or some medication in the previous week. Constipation was defined as fewer than 1 evacuation per day and/or presence of effort during bowel evacuation.

The protocol was approved by the Institutional Review Board of the College of Pharmacy. The study was performed in accordance with the Declaration of Helsinki and amendments relevant to biomedical research involveing human subjects, and the principles of the Guideline for Good Clinical Practice [17,18]. All eligible volunteers were informed of the aim and risks of the study by the clinical researchers and provided written informed consent before enrollment.

\subsection{Administration of Probiotic}

BIO L6 ${ }^{\circledR}$ is a functional food consisting of $10^{8}$ CFUs of lyophilized LAB strains in $10 \mathrm{~g}$ of a powder that is diluted in $250 \mathrm{~mL}$ of water. The probiotic formulations were manufactured and provided by Health-Tech-SigrahZellet S.A. de C.V. (Mexico). Each formulation was analyzed for the number of viable cells and absence of microbiologic contaminants. The genotypic 16S rRNA identification of the organisms used in the present study for the probiotic culture is summarized in Table $\mathbf{1}$.

Each dose was taken upon awakening and diluted in $250 \mathrm{~mL}$ of water. Subjects ingested the probiotic five times a week for two weeks. The treatment was stopped if subjects presented severe side effects or toxicity; e.g., acute diarrhea or fever. If patients presented moderate side effects, the dose was reduced.

\subsection{Evaluations}

Upon enrollment, subjects were asked to record each parameter in a specific case report form throughout the week before each visit: $T_{0}$ : ten days before the beginning of treatment; $\mathrm{T}_{10}$ : ten days during treatment; and $\mathrm{T}_{20}$ : ten days after treatment. In addition to the case report forms, the subjects' general condition and vital status were assessed during each visit. The parameters recorded in the forms were: frequency (number of daily evacuations); ease of expulsion; consistency of feces; flatulence; colic or pain.

Although determining the number of defecations was simple and direct, the other parameters were noted and recorded using the easiest method for the subject, that is, identifying the lowest value with 0 or 1 , the mid-range

Table 1. Lactic acid bacteria contained in Bio $\mathrm{L6}^{\circledR}$ probiotic.

\begin{tabular}{ll}
\hline $\begin{array}{l}\text { Laboratory Strain } \\
\text { Identification }\end{array}$ & $\begin{array}{l}\text { 16S RNA SEQUENCING (FIRST } \\
\text { 500 bp) Microbial ID Inc. }\end{array}$ \\
\hline UA/PHL-LAB 18 & Pediococcus parvulus \\
UA/PHL-LAB 24 & Weissella confusa \\
UA/PHL-LAB 27 & Weissella confusa \\
UA/PHL-LAB 29 & Pediococcus parvulus \\
UA/PHL-LAB 36 & Lactobacillus salivaruis \\
UA/PHL-LAB 37B & Weissella confusa \\
UA/PHL-LAB 40 & Weissella confusa \\
UA/PHL-LAB 44 & Weissella paramesenteroides \\
UA/PHL-LAB 46 & Lactobacillus salivaruis \\
UA/PHL-LAB 48 & Lactobacillus salivaruis \\
UA/PHL-LAB 52 & Pediococcus parvulus \\
\hline
\end{tabular}


with 2 and the highest one with 3 . The value scale was explained and agreed upon with the volunteers as follows:

1) Number of daily evacuations $(<1 \mathrm{y}>1)$.

2) Effort during expulsion: 1, without effort; 2, effort without pain; 3, effort with pain.

3) Consistency of feces: 1 , hard; 2 , soft; 3 , semi-liquid.

4) Flatulence: 0 , none; 1 , little; 2 , regular; 3 , abundant.

5) Pain, colic: 0 , no pain; 1 , light pain; 2 , medium pain; 3 , intense pain.

\subsection{Primary and Secondary Endpoints}

The primary efficacy endpoint was the number of evacuations in $24 \mathrm{~h}$. The secondary efficacy end points were ease of expulsion during defecation and feces consistency during the treatment with the probiotic. All data were prospectively obtained from two questionnaires and registered onto a structured database. Adherence to treatment was monitored through interviews with each patient and the delivery of empty bottles. Subjects who voluntarily discontinued the treatment or forgot to take powder formulations for at least 3 days were considered dropouts.

Probiotics colonization was probed by taking a stool culture of lactobacillus from each patient previous to treatment and in the last day of treatment. The assay allowed us to morphologically characterize the presence of LAB colonies before and after treatment.

\subsection{Statistical Analysis}

The number of daily defecations is expressed as mean \pm SD. The number of evacuations was compared by one way analysis of variance using the GLM procedure of SAS with significance reported as $P<0.05$. The chisquared test of independence was used to determine significant differences $(P<0.001)$ in presence or absence of constipation and feces consistency before and after treatment.

\section{Results}

\subsection{Evaluation of Efficacy}

A total of 30 healthy volunteers were identified. Out of these, 25 subjects (17 females and 8 males) were selected for the present study according to inclusion criteria. One subject withdrew prematurely from treatment because it increased her constipation. 24 healthy volunteers finished the study. The average age was 25.5 years (range 18 - 55 years) and a total of 23 subjects showed some degree of improvement.

Table 2 shows the effect of probiotic Bio L6 ${ }^{\circledR}$ on the number of daily evacuations. As we can see, 23 subjects had an increase in evacuation frequency during treatment
( $p<0.001$ ) (Table 3) and 20 subjects continued to experience increased frequency in excretion 10 days after they had finished treatment $(p<0.001)$ (Table 3).

Table 4 shows the effect of probiotic Bio L6 ${ }^{\circledR}$ on expulsion effort. The number of subjects who experience stool expulsion without effort increased to 16 (66.6\%)

Table 2. Effect of Bio $\mathrm{LG}^{\circledR}$ probiotic consumption of on the number of evacuations.

\begin{tabular}{ccc}
\hline & \multicolumn{3}{c}{ Frequency } \\
\hline & $<1$ & $>1$ \\
$\mathrm{~T}_{0}$ & $100 \%$ & $0.0 \%$ \\
$\mathrm{~T}_{10}$ & $4.1 \%$ & $95.8 \%$ \\
$\mathrm{~T}_{20}$ & $16.6 \%$ & $83.3 \%$ \\
\hline
\end{tabular}

Table 3. Assessment of the main parameters associated to evacuation disorders (Mean \pm SD) before and after the administration of Bio $\mathrm{LG}^{\circledR}$ probiotic.

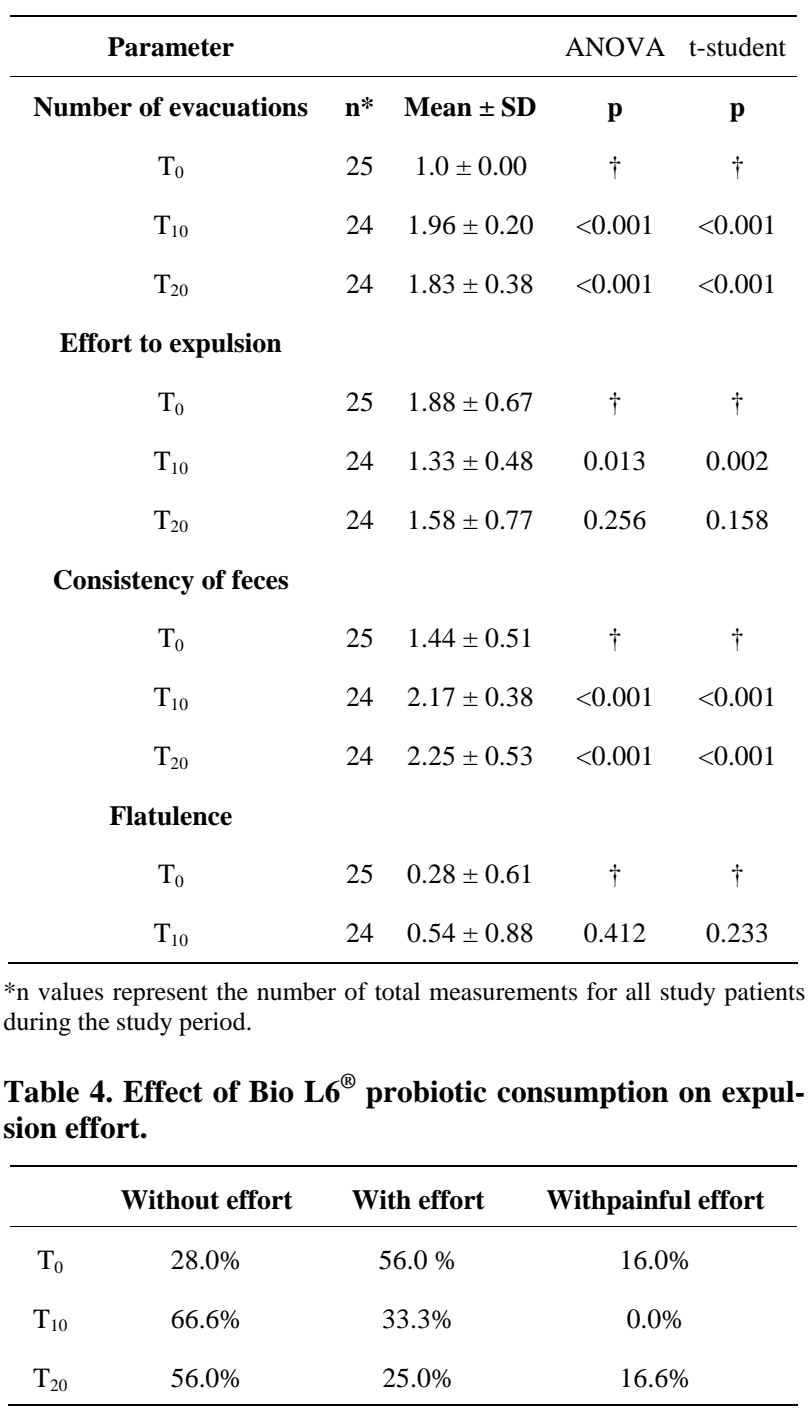


during the treatment, while 14 subjects continued to evacuate effortlessly after finishing treatment $(p<0.001)$ (Table 3). We also observed an important reduction in pain during defecation. 8 subjects $(33.3 \%)(p<0.001)$ experienced effort during expulsion while on the treatment and 6 subjects (25\%) continued to experience this problem after treatment. Previous to treatment, four volunteers experienced painful effort when defecating; all of them had painless evacuations during treatment, but went back to experiencing pain during expulsion after treatment.

Table 5 shows the effect of Bio $L 6^{\circledR}$ probiotic consumption on feces consistency. No one had hard feces during treatment $(0 / 24)$ and just one subject had hard feces after treatment $(p<0.001)$. Twenty subjects $(83.3 \%)$ had soft feces during treatment and only 16 subjects (66.6\%) continued to present soft feces $(p<0.001)$ after treatment. Four subjects showed semi-liquid feces during treatment and seven subjects showed semi-liquid feces after treatment $(p<0.001)$ (Table 3).

\subsection{Evaluation of Safety}

Most treatment-related AEs were considered mild or regular in terms of severity. Symptoms were consistent with AEs previously reported for probiotic consumption and included flatulence, abdominal pain, diarrhea or constipation (Table 6). 13 subjects reported mild AEs and 7 reported regular AEs. The most common were mild flatulence (20.8\%) and regular flatulence (12.5\%). 12.5\% subjects and regular abdominal pain reported mild abdominal pain by $8.3 \%$. Mild diarrhea was reported by $12.5 \%$ of subjects. One subject had severe constipation and withdrew prematurely from treatment; 24 subjects continued taking probiotics; they tolerated probiotic consumption until the end of the follow-up.

Table 5. Effect of Bio $\mathrm{L6}^{\circledR}$ probiotic consumption on feces consistency.

\begin{tabular}{cccc}
\hline Feces consistency & Hard & Soft & Semi-liquid \\
\hline $\mathrm{T}_{0}$ & $54.1 \%$ & $50.0 \%$ & $0.00 \%$ \\
$\mathrm{~T}_{10}$ & $0.0 \%$ & $83.3 \%$ & $16.6 \%$ \\
$\mathrm{~T}_{20}$ & $4.1 \%$ & $66.6 \%$ & $29.1 \%$ \\
\hline
\end{tabular}

Table 6. Adverse events during consumption of Bio $\mathrm{LG}^{\circledR}$ probiotic.

\begin{tabular}{ccc}
\hline Adverse event & Mild & Regular \\
\hline Flatulence & $20.8 \%$ & $12.5 \%$ \\
Abdominal pain & $12.5 \%$ & $8.3 \%$ \\
Diarrhea & $12.5 \%$ & $0.0 \%$ \\
Constipation & $0.0 \%$ & $8.3 \%$ \\
\hline
\end{tabular}

\subsection{Bacteriological Study}

We observed the presence of at least 3 different types of LAB that were present in BIO L6 and absent from volunteers' stool before treatment; these were present in stools during treatment and two days after it in all subjects, meaning that the LAB present in the probiotic carried out intestinal colonization. We also observed a decrease in coliform bacteria (data not shown).

\section{Discussion}

Almost a century ago, Élie Metchnikoff proposed the revolutionary idea of consuming viable bacteria to promote health [19]. The field known as "probiotics" has made dramatic progress since then, particularly during the past two decades. It is known that colonization begins at birth and is followed by the progressive assembly of a complex and dynamic microbial society regulated by elaborate and combinatorial microbial-microbial and hostmicrobial interactions [5]. This microflora has a wide range of functions in the host: it directs the assembly of the gut-associated lymphoid tissue; "educates" the immune system; modulates proliferation and differentiation of its epithelial lineages; regulates angiogenesis; modifies the activity of the enteric nervous system, and plays a key role in extracting and processing nutrients consumed in the diet $[4,20-23]$. The fragile composition of gut microflora can be affected by various factors such as age, diet, environment, stress and medication. Changes in microflora will in turn affect the physiology and pathophysiology of GIT, particularly in the human intestine [24]. The aim of this prospective study with historical control was to evaluate the efficacy and safety of a probiotic mixture in healthy volunteers with evacuation disorders, a very frequent problem in healthy populations of any age and one with important economic and social implications.

The motility of the GIT is important for absorption, transport, and clearance. Absorption is promoted by slow transit because of prolonged contact time, whereas clearance is aided by rapid transit [25]. The effect of gut microflora on intestinal motility takes place through several known mechanisms, including the release of substances that stimulate the enteric nervous system and primary afferent neurons. This process occurs in a context of infection and inflammation as well as in the healthy gut [26]. The present study demonstrates that Bio L6 ${ }^{\circledR}$ increased the number of evacuations in treated subjects and that this effect continued ten days after the end of treatment. Previous studies have shown that gut transit is slow in the absence of intestinal microflora [27]. It has also been reported that intestinal microflora is involved in the development and maintenance of gut sensory and motor functions through the release of bacterial substan- 
ces, fermentation products and intestinal neuroendocrine factors, as well as through a close link with the gastrointestinal immune system [28]. The end products of bacterial metabolism affect gut motor function via neuromodulation and have direct effects on intestinal smooth muscle contractility [29]. The cyclic recurrence and distal propagation of interdigestive migrating motor complexes (MMCs) is also linked to intestinal flora. It has been shown that germ-free animals have delayed gastric emptying and slowed intestinal transit compared with conventionally raised counterparts. It has also been demonstrated that the introduction of gut microflora into germfree rats stimulated interdigestive intestinal motility and accelerated intestinal transit [30,31]. We demonstrated that subjects receiving Bio L6 ${ }^{\circledR}$ experienced increased ease expulsion during and after 10 days of treatment, which indicates that Bio L6 ${ }^{\circledR}$ accelerated intestinal transit in these subjects.

Constipation means different things to different individuals; some think of it as stool frequency, others relate it to stool form and consistency, difficulties defecating or a sense of incomplete evacuation [32]. A number of studies have demonstrated probiotics' ability to shorten colonic transit and soften the feces in healthy subjects, the elderly, and people with irritable bowel syndrome (IBS) [33-35]. Our research agrees with those studies: subjects showed an important softening of feces during and after Bio L6 ${ }^{\circledR}$ treatment.

The safety of the microorganisms that have been traditionally used in probiotics has been confirmed through a long period of experience. Side effects are rare, but the most common is gastrointestinal distress. When large doses of probiotics are consumed, they adjust the floral balance of the digestive tract and can result in gas or abdominal discomfort. These side effects are usually temporary and, ultimately, benign [36,37]. In present study, reported side effects encompassed flatulence, abdominal pain and diarrhea.

This pilot study showed that a probiotic mixture containing eleven strains of LAB (Bio L6 ${ }^{\circledR}$ ) was effective, safe and beneficial in subjects suffering from evacuation disorders. Some of the positive effects continued for 10 days after the suspension of the active formulations. This was due to an active reproduction of the strains in the intestinal lumen, as demonstrated by the microbiological studies.

\section{Acknowledgements}

We would like to thank the Arkansas Biosciences Institute Supported Research project entitled "Development of a Lactobacillus Probiotic for Reducing Cancer-Associated Immunosuppressive and Antibiotic-Induced Diarrhea”.

\section{REFERENCES}

[1] P. B. Eckburg, E. M. Bik, C. N. Bernstein, E. Purdom, L. Dethlefsen, M. Sargent, S. R. Gill and K. E. Nelson, "Diversity of the Human Intestinal Microbial Flora,” Science, Vol. 308, No. 5728, 2005, pp. 1635-1638. doi:10.1126/science.1110591

[2] Å. Sullivan and C. E. Nord, "Probiotics and Gastrointestinal Diseases,” Journal of Internal Medicine, Vol. 257, No. 1, 2005, pp. 78-92. doi:10.1111/j.1365-2796.2004.01410.x

[3] A. Suau, R. Bonnet, M. Sutren, J. J. Godon, G. R. Gibson, M. D. Collins and J. Doré, "Direct Analysis of Genes Encoding 16 s Rrna from Complex Communities Reveals Many Novel Molecular Species within the Human Gut," Applied Environmental Microbiology, Vol. 65, No. 11, 1999, pp. 4799-4807.

[4] E. F. Verdu and S. M. Collins, "Microbial-Gut Interactions in Health and Disease. Irritable Bowel Syndrome," Best Practice in Research of Clinical Gastroenterology, Vol. 18, No. 2, 2004, pp. 315-321. doi:10.1016/j.bpg.2003.11.003

[5] R. A. Rastall, "Bacteria in the Gut: Friends and Foes and How to Alter the Balance,” Journal of Nutrition, Vol. 134, No. 8, 2004, pp. 2022S-2026S.

[6] E. F. Verdú, P. Bercík, G. E. Bergonzelli, X. X. Huang, P. Blennerhasset, F. Rochat, M. Fiaux, R. Mansourian, I. Corthésy-Theulaz and S. M. Collins, "Lactobacillus Paracasei Normalizes Muscle Hypercontractility in a Murine Model of Postinfective Gut Dysfunction,” Gastroenterology, Vol. 127, No. 3, 2004, pp. 826-837. doi:10.1053/j.gastro.2004.06.007

[7] V. Gupta and R. Garg, "Probiotics," Indian Journal of Medical Microbiology, Vol. 27, No. 3, 2009, pp. 202-209. doi:10.4103/0255-0857.53201

[8] S. Santosa, E. Farnworth and P. J. Jones, "Probiotics and Their Potential Health Claims,” Nutrition, Vol. 64, No 6, 2006, pp. 265-274.

[9] M. Del Piano, L. Morelli, G. P. Strozzi, S. Allesina, M. Barba, F. Deidda, P. Lorenzini, M. Ballaré, F. Montino, M. Orsello, M. Sartori, E. Garello, S. Carmagnola, M. Pagliarulo and L. Capurso, "Probiotics: From Research to Consumer,” Digestive Liver Disease, Vol. 38, No. 2, 2006, pp. S248-S255. doi:10.1016/S1590-8658(07)60004-8

[10] Y. Benno, F. He, M. Hosoda and H. Hashimoto, "Effect of Lactobacillus GG Yoghurt on Human Intestinal Microecology in Japanese Subjects,” Nutrition Today, Vol. 31, No. 1, 1996, pp. 9S-11S. doi:10.1097/00017285-199611001-00004

[11] M. A. López Cara, P. J. Tárraga López, M. Cerdán Oliver, J. M. Ocaña López, A. Celada Rodríguez, J. Solera Albero and M. A. Palomino Medina, "Constipation in the Population over 50 Years of Age in Albacete Province," Revista Española de Enfermedades Digestivas, Vol. 98, No. 6, 2006, pp. 449-459.

[12] M. E. Sanders, "Effect of Consumption of Lactic Cultures on Human Health,” Adverse Food Nutrition, Vol. 37, 1993, pp. 67-130. doi:10.1016/S1043-4526(08)60116-3

[13] R. Spiller, "Review Article: Probiotics and Prebiotics in 
Irritable Bowel," Alimentary Pharmacology \& Therapeutics, Vol. 28, No. 4, 2008, pp. 385-396. doi:10.1111/j.1365-2036.2008.03750.x

[14] M. Pimentel and C. Chang, "Inflammation and Microflora," Gastroenterology Clinical of North American, Vol. 40, No. 1, 2011, pp. 69-85. doi:10.1016/j.gtc.2010.12.010

[15] M. Othman, R. Agüero and H. C. Lin, "Alterations in Intestinal Microbial Flora and Human Disease,” Current Opinion in Gastroenterology, Vol. 24, No. 1, 2008, pp. 11-16. doi:10.1097/MOG.0b013e3282f2b0d7

[16] W. H. Ling, O. Hanninen and H. Mykkanen, "Colonization and Fecal Enzyme Activities after Oral Lactobacilus GG Administration in Elderly Nursing Home Residents,” Annual Nutrition and Metabolism, Vol. 36, No. 3, 1992, pp. 162-166. doi:10.1159/000177712

[17] World Medical Association Declaration of Helsinki, "Recommendations Guiding Medical Doctors in Biomedical Research Involving Human Subjects,” Edinburgh, WMA, 2000. http://www.wma.net

[18] European Agency for the Evaluation of Medicinal Products, International Conference on Harmonization-World Health Organization, "Guideline for Good Clinical Practice,” ICH Topic E6 Geneva, 2002.

http://www.emea.eu.int

[19] I. Metchnikoff, "Prolongation of Life: Optimistic Studies,” Putnam \& Sons, New York, 1908.

[20] J. H. Cummings, J. L. Rombeau and T. Sakata, "Physiological and Clinical Aspects of Short-Chain Fatty Acids,” Cambridge University Press, Cambridge, 1995.

[21] H. Mahrous, "Probiotics Bacteria from Egyptian Infants Cause Cholesterol Removal in Media and Survive in Yoghurt,” Food and Nutrition Sciences, Vol. 2, No. 2, 2011, pp. 150-155. doi:10.4236/fns.2011.22021

[22] C. F. Favier, E. E. Vaughan, M. W. De Vos and A. D. Akkermans, "Molecular Monitoring of Succession of Bacterial Communities in Human Neonates," Applied of Environmental Microbiology, Vol. 68, No. 1, 2002, pp. 219226. doi:10.1128/AEM.68.1.219-226.2002

[23] J. J. Cebra, "Influences of Microbiota on Intestinal Immune System Development,” American Journal Clinical Nutrition, Vol. 69, No. 5, 1999, pp. 1046S-1051S.

[24] S. L. Gorbach "Perturbation of Intestinal Microflora," Veterinary and Human Toxicology, Vol. 35, No. 1, 1993, pp. $15-23$.

[25] E. M. Wright, M. G. Martin and E. Turk, "Intestinal Absorption in Health and Disease-Sugars," Best Practice Research of Clinical Gastroenterology, Vol. 17, No. 6, 2003, p. 943. doi:10.1016/S1521-6918(03)00107-0

[26] G. D. Abrams and J. E. Bishop, "Effect of the Normal Microbial Flora on Gastrointestinal Motility,” Proceeding Society of Experimental Biological Medicine, Vol. 126, No. 1, 1967, pp. 301-304.
[27] L. V. Hooper, T. Midtvedt and J. I. Gordon, "How HostMicrobial Interactions Shape the Nutrient Environment of the Mammalian Intestine," Annual Review of Nutrition, Vol. 22, 2002, pp. 283-307. doi:10.1146/annurev.nutr.22.011602.092259

[28] V. Lesniewska, I. Rowland, H. N. Laerke, G. Grant and P. J. Naughton, "Relationship between Dietary-Induced Changes in Intestinal Commensal Microflora and Duodenojejunal Myoelectric Activity Monitored by Radiotelemetry in the Rat in Vivo,” Experimental Physiology, Vol. 91, No. 1, 2006, pp. 229-237. doi:10.1113/expphysiol.2005.031708

[29] C. Cherbut, A. C. Aube, H. M. Blottiere and J. P. Galmiche, "Effects of Short Chain Fatty Acids on Gastrointestinal Motility,” Scandinavian Journal Gastroenterology, Vol. 222, 1997, pp. 58-61.

[30] E. Husebye, P. M. Hellström, F. Sundler, J. Chen and T. Midtvedt, "Influence of Microbial Species on Small Intestinal Myoelectric Activity and Transit in Germ-Free Rats," American Journal of Physiology and Gastrointestinal Liver Physiology, Vol. 280, No. 3, 2001, pp. G368G380.

[31] E. Husebye, P. M. Hellstrom and T. Midtvedt, "Introduction of Conventional Microbial Flora to Germfree Rats Increases the Frequency of Migrating Myoelectric Complexes,” Journal Gastrointestinal Motility, Vol. 4, No. 1, 1992, pp. 39-45. doi:10.1111/j.1365-2982.1992.tb00077.x

[32] M. M. Tabbers, I. De Milliano, M. G. Roseboom and M. A. Benninga, "Is Bifidobacteriumbreve Effective in the Treatment of Childhood Constipation? Results from a Pilot Study,” Nutrition Journal, Vol. 10, No. 19, 2011, pp. 1-5.

[33] Y. Vandenplas and M. Benninga, "Probiotics and Functional Gastrointestinal Disorders in Children,” Journal of Pediatric Gastroenterology Nutrition, Vol. 48, Suppl. 2, 2009, pp. S107-S109. doi:10.1097/MPG.0b013e3181a1603a

[34] A. Chmielewska and H. Szajewska, "Systematic Review of Randomised Controlled Trials: Probiotics for Functional Constipation,” World Journal of Gastroenterology, Vol. 16, No. 1, 2010, pp. 69-75.

[35] H. Zaharoni, E. Rimon, H. Vardi, M. Friger, A. Bolotin and D. R. Shahar, "Probiotics Improve Bowel Movements in Hospitalized Elderly Patients-The PROAGE Study," Journal of Nutrition and Health Aging, Vol. 15, No. 3, 2011, pp. 215-220. doi:10.1007/s12603-010-0323-3

[36] N. Ishibashi and S. Yamazaki, "Probiotics and Safety," American Journal of Clinical Nutrition, Vol. 73, No. 2, 2001, pp. 465S-470S.

[37] Saxelin, K. Collins, G. Mogensen, S. Birkeland and T. Mattila-Sandholm, "Demonstration of Safety of Probiotics: A Review,” International Journal of Food Microbiology, Vol. 44, No. 1-2, 1998, pp. 93-106. doi:10.1016/S0168-1605(98)00128-7 